\section{$\mathbb{T}$ Periodica Polytechnica Civil Engineering}

OnlineFirst (2018) paper 11961

https://doi.org/10.3311/PPci.11961

Creative Commons Attribution (i)

\title{
Experimental Evaluation of Fragments from TBM Disc Cutting under Different Load Cases
}

\author{
Yimin $\mathrm{Xia}^{1,2}$, Yupeng Shi ${ }^{1,2}$, Laikuang Lin ${ }^{1,2, *}$, Yichao Zhang ${ }^{3}$, \\ Qing Tan ${ }^{1,2}$, Yang Yang ${ }^{1,2}$ \\ Received 15 January 2018; Accepted 18 February 2018
}

\begin{abstract}
The Tunnel Boring Machine (TBM) tunneling process always contains a certain degree of vibrations due to the step broken phenomenon of the cutting tools. Undoubtedly, there is a quite difference in the fragment characteristics which are related to the construction efficiency of TBM under the static load and the combination of static and impact load. In this study, a series of rock breaking tests with a $216 \mathrm{~mm}$ diameter disc cutter and marble samples were conducted under different load cases. Based on the Rosin-Rammler distribution curve, the fragments from the cutting tests were also sieved to calculate the absolute size constant $\left(x^{\prime}\right)$ and coarseness index (CI). The relationship between coarseness index, absolute size parameter and the cutting parameters, specific energy, production rate was evaluated. The results show that there is an increasing trend of $x^{\prime}$ and $C I$ with the increase of cut spacing and penetration as well as adding impact load component. An overall downtrend in specific energy and upward trend in production rate which are associated with the high efficiency can be observed with the increasing CI and $x^{\prime}$. It is believed that the conclusions are of great significance for improving TBM construction efficiency and cutterhead design.
\end{abstract}

\section{Keywords}

disc cutter, fragments characteristics, impact load, absolute size constant, coarseness index

\footnotetext{
1 College of Mechanical and Electrical Engineering,

Central South University, Changsha, 410083, China

2 State Key Laboratory of High Performance Complex Manufacturing,

Central South University, Changsha, 410083, China

3 Patent Examination Cooperation Sichuan Center of the Patent Office, SIPO,

Chengdu, 610000, China

* Corresponding author, email: linlaikuang34@163.com
}

\section{Introduction}

Due to the high efficiency, reliability and economy, the tunnel boring machine (TBM) is widely applied in the tunnel excavations, water conservancy projects and other constructions [1, 2]. As a key component in the TBM, disc cutter achieves the destruction and peeling of rock from tunnel face, which is directly related to the TBM's performance. On the one hand, owing to the complexity of geological conditions, TBMs need to be applicable for various grounds such as rock, soil and some mixed grounds [3]. In the conditions of high geostress, there will be some obvious step broken phenomenon and corresponding vibrations on the disc cutters in the process of breaking rock, resulting in the impact load. On the other hand, the rock breaking mechanism will change under the impact load. Therefore, there are also a lot of ways to actively apply the impact load in the tunnel constructions and mineral mining like split Hopkinson pressure bar (SHPB) and drop hammer. There is strong evidence that the fragments and energy efficiency are quite different between static load and combination load [4]. Thus, it is of great significance for the high efficiency and safe construction of TBM to study on the performance under static load and combination of static and impact load.

Considering the harsh field conditions during TBM construction and the advantages of low cost, repeatability and easy operability of numerical simulation and indoor experiments, many scholars in the United States, South Korea, Turkey, China and other countries successively developed TBM cutter performance test benches. And the rock breaking mechanism of disc cutter has been studied thoroughly and comprehensively [5-7]. Cho et al. [8-9] conducted a series of rock breaking experiments using linear-cutting-machine and recorded the cutting forces and the volume of fragments. The best combination of cut spacing and penetration was deduced according to the specific energy. Zaré and Mikaeil [10] applied the discrete element method to simulate the process of penetrating the jointed rock by two TBM disc cutters. The cut spacing was optimized on the basis of chip thickness, chipping area and penetration rate. The mechanical properties of rock samples under impact load were also studied in numerous 
references. Li et al. [11], Gong et al. [12], Zhu et al. [13] carried out the single-axis static and impact load tests for different kinds of rock samples through low-cycle-index fatigue loading method. It can be found that on the basis of static load, the stiffness of the rock will be reduced by applying impact load. The rock fragmentation develops to micro particles. Zhao et al. [14-16] introduced a new testing device to break rock under the combination of static and impact load and presented a laboratory test. The results show that combination load can promote the formation of fragments. The impact load with high energy and frequency is beneficial to the cutting of brittle hard rock. Based on the experimental studies, Dyskin et al. [17] and $\mathrm{Li}$ et al. [18] found that applying the combination load can improve the energy acting on the rock and promote the development of cracks, which is an efficient method to break rock. For the cases that the compressive strength of rock surface was extremely high, the low excavation speed and serious abrasion could be expected. Rostami et al. [19] believed taking advantage of impact load to break rock in hard rock TBM engineering might conclude a better effect.

Coarseness index $(C I)$, which is the sum of the cumulative mass percentages of broken rock in a particular size screening device, is regarded as one of the effective indicators for evaluating the efficiency of mechanical excavation. Brezáni and Zeleňák [20] and Sampson [21] analyzed the size distribution of fragmentation collected in linear cutting tests and tunnel engineering sites. The production of large-size crushing blocks has a direct effect on rock breaking efficiency and energy consumption. Roxborough et al. [22] calculated the $C I$ values of fragments from disc cutting experiments on the saturated and reported an inverse trend between specific energy and coarseness index for chalk fragments. Besides, the calculated coarseness index values for saturated samples are always lower than that for the dry samples. Bakar et al. [23] evaluated fragments of dry and saturated sandstone generated from disc cutting tests and held the same conclusions as Roxborough. Altindag [24-25], Kahraman et al. [26], Gertsch et al. [27] also found a certain correlation between $C I$ values and the penetration rates of drills in rock drillability studies. Jung et al. [28] adopted $C I$ to evaluate the fragments generated from a tunnel face in a TBM tunnel construction and reported an increasing trend in the $C I$ values with the increase of penetration from 2 to $4 \mathrm{~mm}$ at the same cut spacing.

As can be seen from the above, a great deal of researches has been done on the analysis of rock breaking mechanism and fragments of disc cutters. However, few literatures are concerned about difference in the fragment characteristics which are related to the construction efficiency of TBM under the static and combination load. In this paper, based on the self-designed TBM cutter performance test bench, the fragmentation characteristics and cutting efficiency of disc cutter under different load cases are analyzed. The relations between cutting parameters, specific energy, production rate and the absolute size parameter, coarseness index are also studied. Considering the capacity of the equipment, the strength of rock samples is not much hard and the main concern of this study is the different effects of static load and combination load applied on disc cutter.

\section{Background}

During TBM construction process, the formation of fragments is closely related to the generation and development of cracks in the rock. Under the normal thrust of the cutterhead, the disc cutters penetrate into the rock and a high-pressure zone inside the rock is generated. According to fracture mechanics theories, when the stress intensity exceeds the critical stress intensity factor of the rock, the cracks will propagate in the direction of the least surface energy, which means that the cracks can be expanded in any direction. Therefore, there are three cutting models which are underbreak cutting model, overbreak cutting model, relieved cutting model depending on the interaction of the cracks between adjacent cutting paths [29]. As shown in Fig. 1(a), the cracks under the disc cutters with large

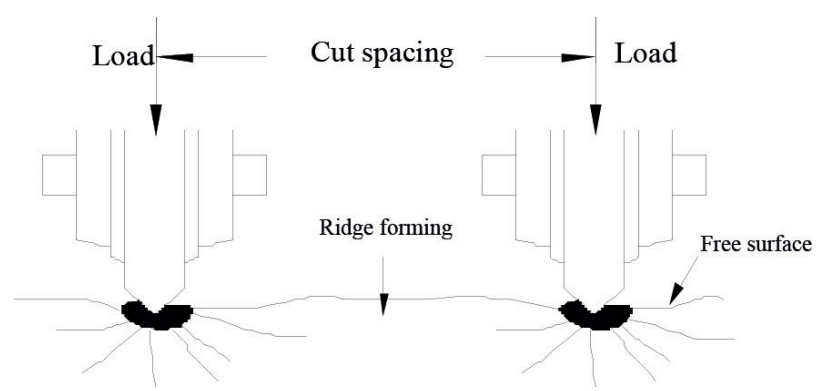

(a) underbreak cutting model

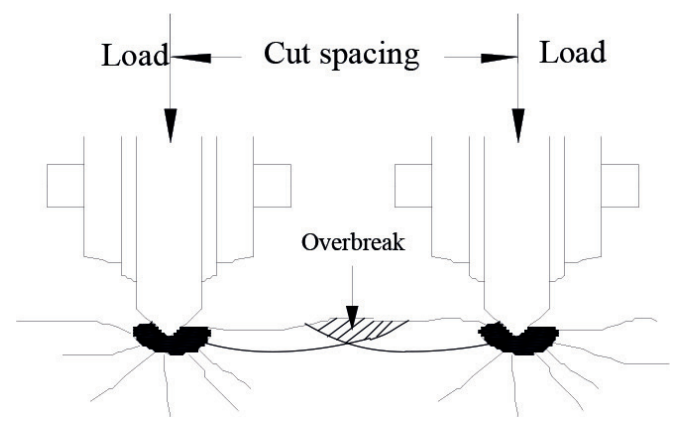

(b) overbreak cutting model

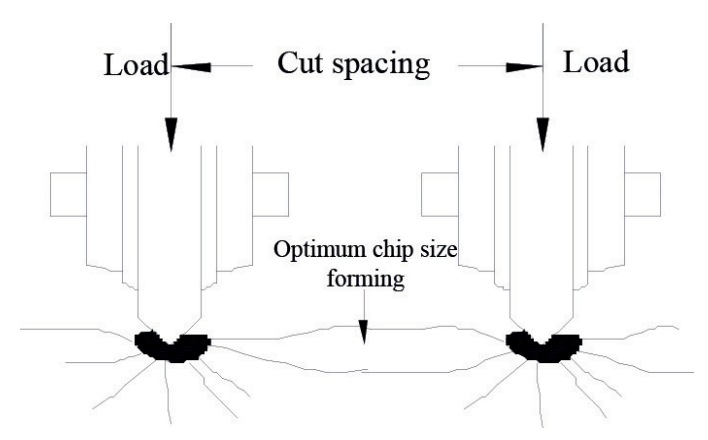

(c) relieved cutting model

Fig. 1 Diagram of three cutting models 
cut spacing cannot intersect and propagate to the free surface, resulting in triangular fragments and the formation of ridges, which is called underbreak cutting model. When the cut spacing is small or the normal thrust increases substantially, the cracks between adjacent cutting paths are staggered and overlapped. It is named as the overbreak cutting model considering that the rock is broken many times, as shown in Fig. 1(b). It can be inferred that there is a critical condition where the cracks between adjacent cutting paths meet in a straight line and each propagates half of the cut spacing, resulting in the formation of a large strip chips. Fig. 1(c) shows the most efficient cutting model, called the relieved cutting model.

The above analysis is based on homogeneous rock media. The initial damage and joints existing in the rock can also affect the model of crack propagation and the formation of fragments.

\section{Experimental details}

\subsection{Test equipment}

In this study, the disc cutting tests were performed on the multifunctional TBM cutter performance test bench at the State Key Laboratory of High Performance Complex Manufacturing (Central South University, CSU). The test bench is composed of three systems which are for vertical static load (VSLS), disturbance load (DLS) and rolling cut (RCS) respectively. The VSLS is made up of vertical hydraulic cylinder and steel frame for loadbearing. The vertical-active crossbeam and the mechanical lock device are to ensure a constant penetration during cutting. The basic design of the RCS is a platform that allows the cutting tool to move freely in the horizontal plane. The rock sample to be cut is cast into a pre-prepared steel tank with high strength concrete and then the tank is placed on the horizontal table just below the cutting tools. The average cutting velocity of the cutter for the tests is set to $40 \mathrm{~mm} / \mathrm{s}$. The cut spacing can be adjusted by moving the horizontal platform. A structural diagram and physical map of multifunctional TBM cutter performance test bench are shown in Fig. 2 and Fig. 3 respectively.

Moreover, the most important component in this study is the DLS which is used to provide the impact load. DLS is installed between the vertical-active crossbeam and a three-directional load cell. It's mainly a vertical guide rod droved by disturbance hydraulic device. Buffer springs are designed to achieve the guide rod automatically reset. The main principle of the impact load is to push the guide rod by adding pressure regularly through hydraulic system to hydraulic cylinders, and eventually passing impact load to the disc cutter, as shown in Fig. 4.

Fig. 5 shows the forces acting on the disk cutter. The normal, rolling and side forces are measured by using a three-directional (3-D) force sensor mounted between the bottom of DLS and the cutter mount. The maximum loading capacity in the direction of cutter thrust is 12 tons $(120 \mathrm{kN})$. A computer-based data acquisition system (including 3-D force sensor, data acquisition card, voltage-stabilized source, computer and the National Instruments
LabVIEW, 2012) is used to record the outputs from the 3-D force sensor. Before the experiments, the 3-D force sensor has been calibrated to ensure the accuracy of the measurement results.

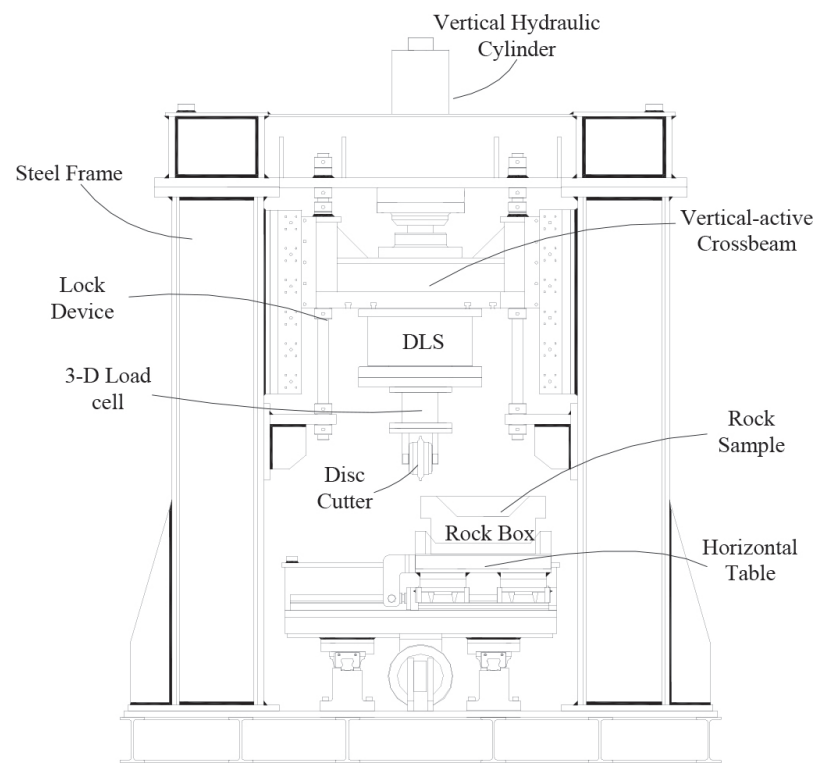

Fig. 2 Schematic view of multifunctional TBM cutter performance test bench at Central South University

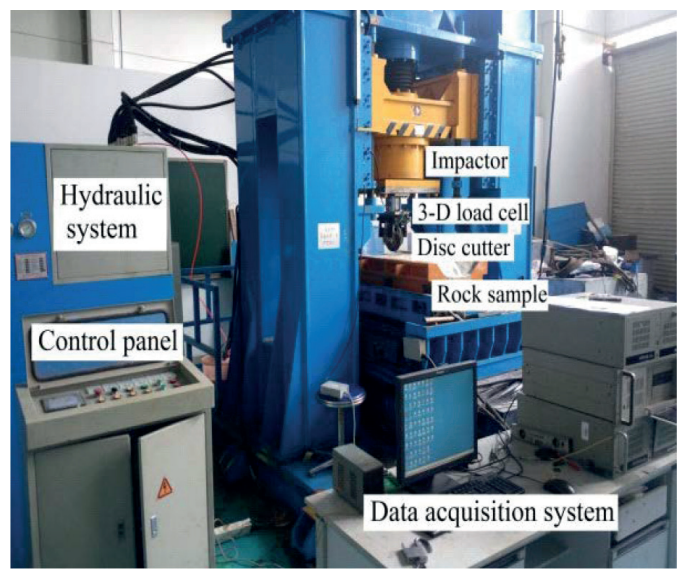

Fig. 3 General view of multifunctional TBM cutter performance test bench with $216 \mathrm{~mm}$ diameter disc cutter at CSU

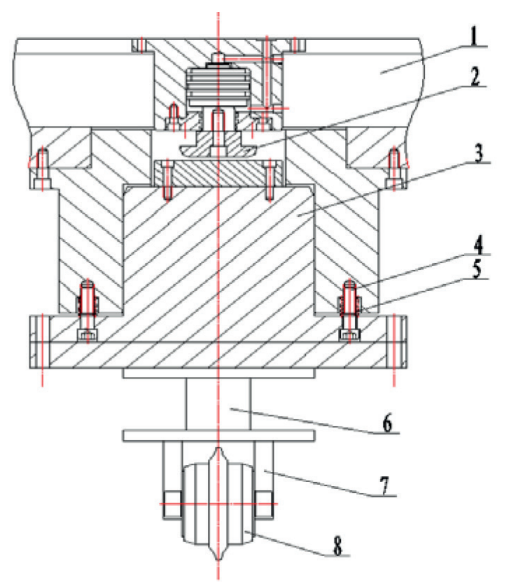

Fig. 4 The principle diagram of the mechanical impactor 1- Vertical-active crossbeam; 2- The piston rod of the impact cylinder; 3 - The guide rod of the impact cylinder; 4- Lock screw; 5- Buffer spring; 6- Three directional load cell; 7- Cutter mount; 8- Disc cutter 


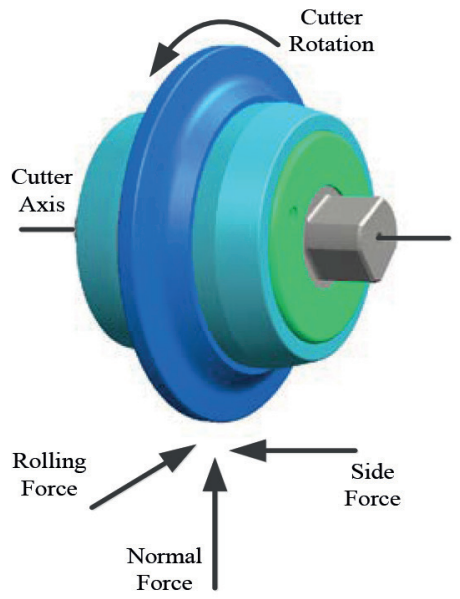

Fig. 5 Forces acting on the disc cutter

\subsection{Disc cutter and rock sample}

Due to the limits of the force sensor, the diameter and tip width of the disc cutter used in tests are $216 \mathrm{~mm}$ and $9.5 \mathrm{~mm}$, respectively. It is the reduction scale of 1:2 of the 17 inches constant cross-section disc cutter.

The marble samples cut in the experiments were obtained from a tunnel engineering site. The marble samples were split into cuboids with the size of $900 * 380 * 260 \mathrm{~mm}$ and then placed in an upper opening iron box with concrete. According to concrete curing standards, it needs at least 21 days for concrete to cure before testing. The physical properties gotten through the mechanical tests of the standard samples are listed in Table 1.

Table 1 Physical properties of test marble

\begin{tabular}{lc}
\hline Properties of marble samples & Value \\
\hline Density & $2580 \mathrm{~kg} / \mathrm{m}^{3}$ \\
Uniaxial compressive strength & $24.92 \mathrm{Mpa}$ \\
Brazilian tensile strength & $4.72 \mathrm{Mpa}$ \\
Poisson's ratio & 0.21 \\
Young's modulus & $7.12 \mathrm{Gpa}$ \\
\hline
\end{tabular}

\subsection{Experimental procedures}

Fig. 6 illustrates test-related terms, showing that rock is divided into several data layers according to different penetrations. Every data layer consists of a few of data windows with the same penetration and different spacing, and each data window is composed of a number of cuts, whose spacing is also called the cut spacing. Based on the cutting paths designed on the surface of the rock sample, the data window was marked on the rock sample surface for the collection of fragments and data acquisition, as shown in Fig.7. It should be noted that the data window does not include data cuts that are closer than one cut spacing to any block boundaries to minimize the adverse effects of the boundary effect on the experimental data. The number of cutting paths in a data window was related to the cut spacing and penetration. Table 2 shows the test matrix for both static load and combination load respectively. The disc cutter could reach the pre-set cut spacing and penetration by adjusting the
Table 2 Test matrix for both static load and combination load

\begin{tabular}{lccc}
\hline Condition & $P(\mathrm{~mm})$ & $S(\mathrm{~mm})$ & $S / P$ \\
\hline Case1 & 2 & 14 & 7 \\
Case2 & 2 & 20 & 10 \\
Case3 & 2 & 30 & 15 \\
Case4 & 2 & 40 & 20 \\
Case5 & 2 & 60 & 30 \\
Case6 & 3 & 14 & 4.7 \\
Case7 & 3 & 20 & 6.7 \\
Case8 & 3 & 30 & 10 \\
Case9 & 3 & 40 & 13.3 \\
Case10 & 3 & 60 & 20 \\
Case11 & 4 & 30 & 7.5 \\
Case12 & 4 & 40 & 10 \\
Case13 & 4 & 60 & 15 \\
Case14 & 4 & 100 & 25 \\
Case15 & 6 & 30 & 5 \\
Case16 & 6 & 40 & 6.7 \\
Case17 & 6 & 60 & 10 \\
Case18 & 6 & 100 & 16.7 \\
\hline
\end{tabular}

$S$ - cut spacing; $P$ - cutter penetration; $S / P$ - the ratio of cutter spacing to penetration depth.

vertical-active crossbeam and horizontal platform and then the marble rock samples would be cut through the RCS. When finishing all the cutting paths in a data window, the rock fragments inside the data window would be carefully collected to avoid further damage during the collection process. The broken chips of each experimental group were then sieved and weighed.

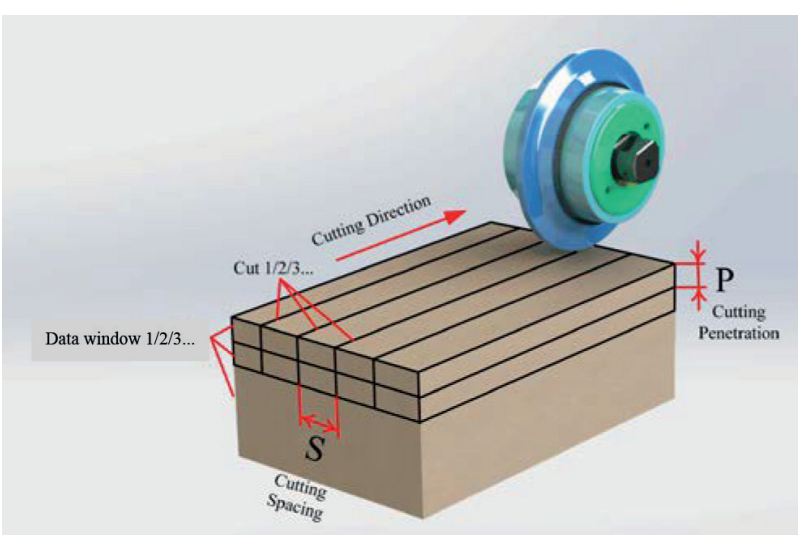

Fig. 6 Test-related terms about the sample

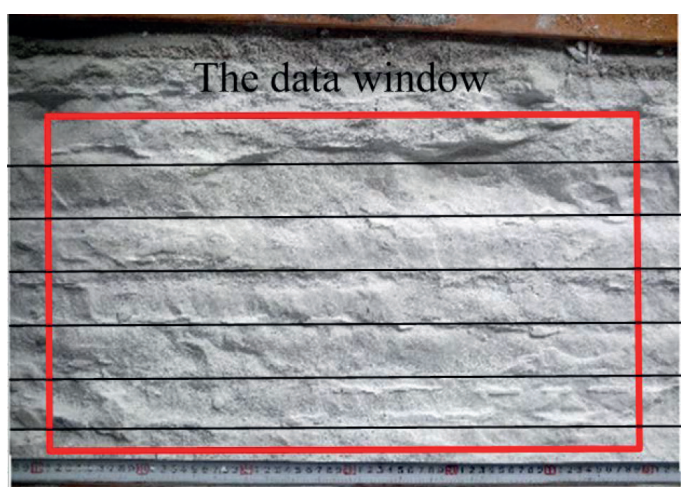

Fig. 7 A data window diagram during the tests 
In this research, the linear cutting machine provided two different load cases, which were static load and combination of static and impact load. During the cutting tests, the impact cylinder was loaded regularly by the hydraulic system, producing an impact load which was eventually delivered to the disc cutter. It was the situation that combination of static and impact load was applied. The magnitude of the impact load could be determined by adjusting the pressure of the hydraulic cylinder. In the experiment, the impact pressure was $20 \mathrm{MPa}$ and the impact load was about $5 \mathrm{kN}$ according to the calibration results. The impact frequency of the system was set to $1 \mathrm{~Hz}$. When the impact pressure was $0 \mathrm{MPa}$, the disc cutter was only applied with static load.

\section{Rock sieve analysis}

After finishing all the cutting paths in a data window, the rock chips and fines were collected with great care to prevent any further breakage of the marble chips and analyzed for particle size distribution. According to the characteristics of the fragmentation, the chips were sieved into seven sizes (larger than 53, 37.5-53, 26.5-37.5, 9.5-26.5, 2.36-9.5, 0.6-2.36 mm and passing $0.6 \mathrm{~mm}$ ), and chips that were oversized on each sieve were weighed separately.

In general, the energy consumed in other directions can be neglected compared with the rolling force on the disc cutter [27]. As one of the most important indicator for the performance evaluation of TBM, specific energy $\left(\mathrm{SE}, \mathrm{J} / \mathrm{m}^{3}\right)$ is calculated as follows:

$$
S E=\frac{W}{V}=\frac{F_{\text {rolling }} \cdot L \cdot \rho}{m}
$$

Where $W$ denotes the average value of work done by rolling force $(\mathrm{J}) ; V$ denotes the volume of the chips $\left(\mathrm{m}^{3}\right) ; F_{\text {rolling }}$ denotes the average rolling force $(\mathrm{N}) ; L$ denotes the cutting distance $(m) ; \rho$ denotes density of marble samples $\left(\mathrm{kg} / \mathrm{m}^{3}\right) ; \mathrm{m}$ denotes the mass of chips $(\mathrm{kg}) . W$ is calculated from the area between the displacement force curve and the abscissa according to the acquisition system, as shown in Fig. 8.

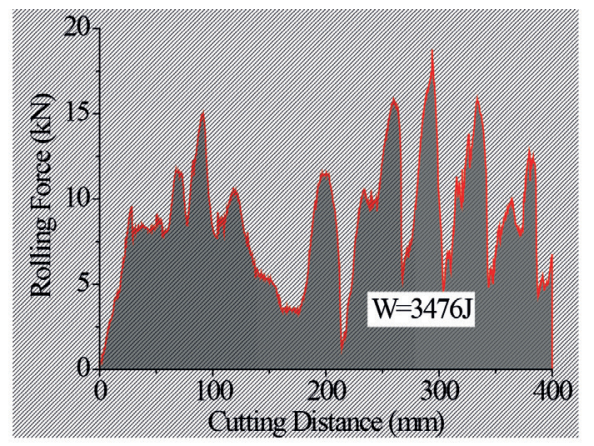

Fig. 8 Example to show the calculation of work done by rolling force

As another very important indicator, production rate $(\mathrm{kg} / \mathrm{min})$ is often applied to predict the performance of the mechanical excavators, too. Production rate includes nominal production rate $\left(P R_{N}\right)$ and actual production rate $\left(P R_{A}\right)$. The actual production rate is calculated from the mass of chips collected from cutting paths and the nominal production rate is the calculated according to the given spacing and penetration without the case of excessive crushing and ridge between the cuts. The formula is as follows:

$$
P R_{N}=s^{*} p * v^{*} \rho
$$

Where $s$ denotes the cut spacing (m); $p$ denotes the cut penetration $(\mathrm{m}) ; v$ denotes the cutting velocity of disc cutter $(\mathrm{m} /$ $\min ) ; \rho$ denotes the density of marble $\left(\mathrm{kg} / \mathrm{m}^{3}\right)$.

Coarseness index is the sum of the cumulative mass percentages of broken rock on every particular sieve. Table 3 gives a typical example of the calculation of $C I$ under the combination load at $s=100 \mathrm{~mm}$ and $p=6 \mathrm{~mm}$. Fig. 9 shows grain size distribution curves by logarithmic rectangular coordinate for the marble sample at the same spacing coupled with different penetrations. It can be found that the proportion of large chips increased with the increase in penetration. This is anticipated because the interaction of adjacent cutting paths will be enhanced.

According to the Rosin-Rammler distribution, the mass distribution function in exponential form is defined as follows:

$$
R=100 e^{\left[-\left(\frac{x}{x^{\prime}}\right)^{b}\right]}
$$

Table 3 Example to calculate the coarseness index under combination load at $s=100 \mathrm{~mm}$ and $p=6 \mathrm{~mm}$

\begin{tabular}{lcc}
\hline Size fraction $(\mathrm{mm})$ & Retained mass $(\mathrm{g})$ & Cumulative mass $(\%)$ \\
\hline+53 & 114.3 & 16.5 \\
$-53+37.5$ & 62.48 & 25.5 \\
$-37.5+26.5$ & 138.41 & 45.5 \\
$-26.5+9.5$ & 76.98 & 56.6 \\
$-9.5+2.36$ & 56.5 & 64.8 \\
$-2.36+0.6$ & 113.66 & 81.2 \\
-0.6 & 130.23 & 100 \\
Total mass & 693.17 & $C I=390.1$ \\
\hline
\end{tabular}

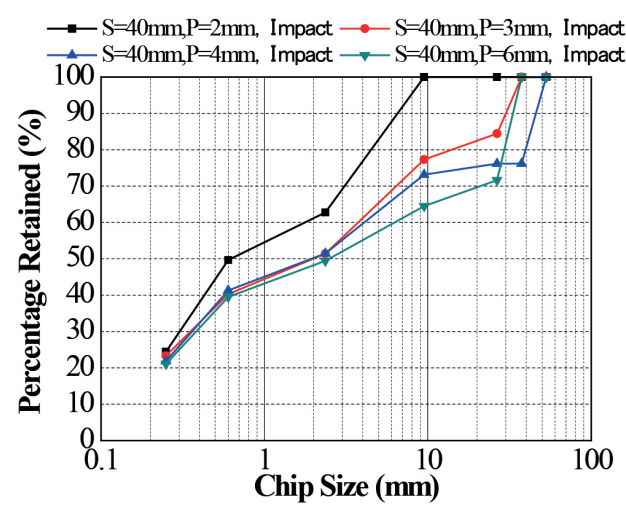

Fig. 9 Size distribution curves at $s=40 \mathrm{~mm}$ and different penetrations under the combination load

Where $R$ denotes the percentage of cumulative mass obtained at every sieve $(\%) ; x$ denotes the size of every sieve $(\mathrm{mm}) ; x^{\prime}$ denotes the absolute size constant $(\mathrm{mm}) ; b$ denotes a constant related to size distribution. 
By equation transformation, Eq. (3) can be written as follows:

$$
\log \left[\log \left(\frac{100}{R}\right)\right]=b \times \log x+\text { cons } \tan t
$$

There is a straight line in the graph of $\log (\log (100 / R))$ versus $\log x$. In the case of $x=x^{\prime}$ in Eq. (4), the $R$ is about $36.79 \%$ [20]. Therefore, $x^{\prime}$ is the abscissa value of the line at $R=36.79 \%$ and represents the most common size of the fragmentation among the distribution. The value of $b$ is the slope of the straight line and reflects the discretization of size distribution. These two parameters reveal the size distribution of fragmentation for a given condition together. Small values of $b$ indicate the wide range of the size distribution, and the larger values of $b$ indicate that the size distribution is narrow [30]. Fig. 10 is an example to show the calculation of $x^{\prime}$ and $b$ for static load at $s=60 \mathrm{~mm}$ and $p=6 \mathrm{~mm}$.

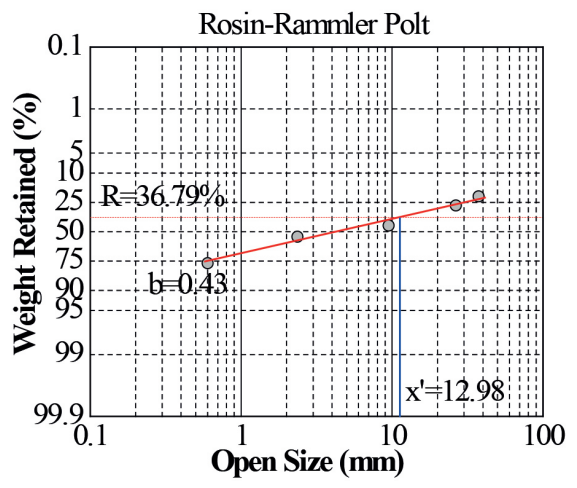

Fig. 10 Determination of absolute size constant $\left(x^{\prime}\right)$ and distribution parameter $b$ using Rosin-Rammler plot for static load at $s=60 \mathrm{~mm}$ and $p=6 \mathrm{~mm}$

\section{Experimental results and discussion}

According to the test matrix, the experimental results, which encompass 4 data layers and 412 cutting paths, are summarized in Table 4 and Table 5. To get the cutting force, energy consumption and other related parameters accurately, the average values are calculated at least from three paths according to the cut spacing. Especially, the actual cutting length of each path is measured. In the case of the same cut spacing, the measured cutting forces appear several irregularities with the increase in penetration. This may be due to the overbreaks of the upper data window, resulting in the required reduction of the forces acting on a disc cutter in the cutting paths of the lower data window. Despite considering the flatness requirements before cutting, because of the irregular distribution of micro-cracks within the rock, this phenomenon is difficult to be avoided. However, the overall trend of experimental data is acceptable to study on the effect of static load and combination load on the degree of fragmentation.

\subsection{Relationship between $x^{\prime}, \mathrm{Cl}$ and cutting parameters}

There is a close relationship between the size of the fragments and cut spacing combined with penetration. The volume of chips increases gradually with cut spacing, reaching a maximum when the mutual influence between the adjacent cutting paths reaches the maximum. Then it decreases to a constant called underbreak cutting model. Fig. 11 and Fig. 12 show the relationship of absolute size constant, coarseness index and cut spacing. Although the data is scattered, it can be observed that

Table 4 Complete test results under static load

\begin{tabular}{|c|c|c|c|c|c|c|c|c|c|}
\hline$S(\mathrm{~mm})$ & $P(\mathrm{~mm})$ & $S / P$ & $F_{N}(\mathrm{kN})$ & $F_{R}(\mathrm{kN})$ & $F_{S}(\mathrm{kN})$ & $C I$ & $S E\left(\mathrm{MJ} / \mathrm{m}^{3}\right)$ & $P R_{A}(\mathrm{~kg} / \mathrm{min})$ & $x^{\prime}(\mathrm{mm})$ \\
\hline 14 & 2 & 7 & 3.89 & 0.42 & 0.48 & 212.0 & 10.15 & 0.13 & 2.44 \\
\hline 14 & 3 & 4.7 & 53.84 & 11.21 & 7.47 & 186.0 & 46.86 & 0.30 & 1.43 \\
\hline 20 & 2 & 10 & 8.20 & 0.79 & 0.32 & 260.2 & 6.84 & 0.36 & 5.32 \\
\hline 20 & 3 & 6.7 & 42.68 & 7.62 & 1.31 & 240.8 & 19.35 & 0.50 & 4.77 \\
\hline 30 & 2 & 15 & 20.61 & 2.75 & 1.37 & 203.6 & 26.85 & 0.32 & 2.07 \\
\hline 30 & 3 & 10 & 44.66 & 8.28 & 7.07 & 365.2 & 36.77 & 1.33 & 20.86 \\
\hline 30 & 4 & 7.5 & 16.08 & 3.13 & 0.39 & 215.7 & 20.67 & 0.47 & 2.58 \\
\hline 30 & 6 & 5 & 32.53 & 9.17 & 2.08 & 209.3 & 26.61 & 0.67 & 2.34 \\
\hline 40 & 2 & 20 & 17.44 & 2.20 & 0.98 & 215.9 & 66.03 & 0.18 & 2.78 \\
\hline 40 & 3 & 13.3 & 61.69 & 11.77 & 9.66 & 246.5 & 46.55 & 1.75 & 10.77 \\
\hline 40 & 4 & 10 & 44.30 & 10.25 & 2.05 & 279.6 & 31.13 & 1.02 & 7.60 \\
\hline 40 & 6 & 6.7 & 48.13 & 11.74 & 7.49 & 298.3 & 24.94 & 1.37 & 8.36 \\
\hline 60 & 2 & 30 & 68.61 & 11.83 & 6.47 & 192.8 & 89.47 & 0.41 & 1.77 \\
\hline 60 & 3 & 20 & 45.38 & 15.91 & 5.00 & 293.8 & 26.54 & 1.86 & 8.21 \\
\hline 60 & 4 & 15 & 66.59 & 13.52 & 2.24 & 484.9 & 14.57 & 2.88 & 38.12 \\
\hline 60 & 6 & 10 & 53.73 & 13.54 & 3.29 & 324.0 & 45.08 & 1.68 & 12.98 \\
\hline 100 & 4 & 25 & 63.32 & 12.89 & 0.82 & 319.4 & 50.29 & 0.75 & 11.32 \\
\hline 100 & 6 & 16.7 & 41.66 & 10.44 & 1.28 & 443.3 & 53.79 & 2.49 & 25.42 \\
\hline
\end{tabular}

$S$ - cut spacing; $P$ - cutter penetration; $F_{N}$ - mean normal force; $F_{R}$ - mean rolling force; $F_{S}$ - mean side force; $C I$ - coarseness index; $S E$ - specific energy; $P R_{A}$ - actual production rate; $x$ - absolute size constant. 
Table 5 Complete test results under combination load

\begin{tabular}{|c|c|c|c|c|c|c|c|c|c|}
\hline$S(\mathrm{~mm})$ & $P(\mathrm{~mm})$ & $S / P$ & $F_{N}(\mathrm{kN})$ & $F_{R}(\mathrm{kN})$ & $F_{S}(\mathrm{kN})$ & $C I$ & $S E\left(\mathrm{MJ} / \mathrm{m}^{3}\right)$ & $P R_{A}(\mathrm{~kg} / \mathrm{min})$ & $x^{\prime}(\mathrm{mm})$ \\
\hline 14 & 2 & 7 & 18.49 & 2.11 & 0.99 & 287.2 & 96.01 & 0.33 & 17.08 \\
\hline 14 & 3 & 4.7 & 35.98 & 8.52 & 1.84 & 212.5 & 51.84 & 0.48 & 2.46 \\
\hline 20 & 2 & 10 & 25.79 & 3.29 & 1.13 & 305.5 & 75.03 & 0.47 & 10.93 \\
\hline 20 & 3 & 6.7 & 42.35 & 11.35 & 4.22 & 262.5 & 38.57 & 0.69 & 5.11 \\
\hline 30 & 2 & 15 & 29.37 & 7.53 & 0.56 & 226.4 & 36.75 & 0.70 & 3.36 \\
\hline 30 & 3 & 10 & 40.13 & 10.87 & 7.97 & 225.1 & 22.44 & 0.87 & 2.90 \\
\hline 30 & 4 & 7.5 & 33.08 & 8.22 & 4.52 & 277.6 & 17.72 & 1.10 & 6.23 \\
\hline 30 & 6 & 5 & 40.67 & 12.44 & 7.19 & 222.4 & 35.06 & 1.10 & 3.11 \\
\hline 40 & 2 & 20 & 20.99 & 5.38 & 0.81 & 263.2 & 32.81 & 0.51 & 8.02 \\
\hline 40 & 3 & 13.3 & 50.38 & 11.05 & 10.4 & 323.3 & 21.57 & 1.59 & 11.88 \\
\hline 40 & 4 & 10 & 53.38 & 12.29 & 4.02 & 359.9 & 25.46 & 1.50 & 16.72 \\
\hline 40 & 6 & 6.7 & 54.42 & 18.29 & 9.63 & 365.9 & 27.21 & 2.10 & 16.97 \\
\hline 60 & 2 & 30 & 4.54 & 0.49 & 0.38 & 164.1 & 67.45 & 0.20 & 0.92 \\
\hline 60 & 3 & 20 & 41.77 & 17.94 & 8.87 & 297.0 & 20.56 & 2.70 & 8.26 \\
\hline 60 & 4 & 15 & 45.32 & 11.96 & 3.84 & 468.2 & 7.9 & 1.61 & 33.97 \\
\hline 60 & 6 & 10 & 39.53 & 12.25 & 3.30 & 373.3 & 13.18 & 1.47 & 29.48 \\
\hline 100 & 4 & 25 & 58.43 & 13.09 & 3.13 & 400.3 & 16.19 & 2.51 & 27.64 \\
\hline 100 & 6 & 16.7 & 41.75 & 10.22 & 0.38 & 390.1 & 15.22 & 2.08 & 23.00 \\
\hline
\end{tabular}

$S$ - cut spacing; $P$ - cutter penetration; $F_{N}$ - mean normal force; $F_{R}$ - mean rolling force; $F_{S}$ - mean side force; $C I$ - coarseness index; $S E$ - specific energy; $P R_{A}$ - actual production rate; $x^{\prime}$ - absolute size constant.

with the increase of cut spacing, absolute size constant and coarseness index have an increasing trend. Different points with the same abscissa values indicate the values at different penetrations. From the comparison, it can be found the values of $x^{\prime}$ and $C I$ generally increase by increasing the penetration. Owing to the increase in penetration, the interaction of lateral cracks between adjacent cutting paths is enhanced, resulting in a large number of big size chips. Under the impact load of the cutter, the interaction will be further strengthened, which leads to the larger chips under the condition of relatively static load. It can be conducted that adding the impact load component can get the same cutting model as the static load at narrower cutting spacing. It will be a practical way to reduce the number of cutting tools on the cutterhead, which indicates lower construction costs. However, the rock ridges will be formed between the two adjacent cutting paths at shallow penetration coupled with large cut spacing. Lack of interconnection of lateral cracks in adjacent cutting paths results in the occurrence of underbreak cutting model. In this case, the fragmentation is almost fine powder, so that the $x^{\prime}$ and $C I$ values are small. This phenomenon can be easily found from rock breaking experiments. Fig. 13 shows a typical underbreak cutting model at large $s / p$ ratios under the combination load before chip collection. Small values of $x^{\prime}$ and $C I$ also appear in the case of small cut spacing due to overbreak cutting model, and the fragmentation is very small.

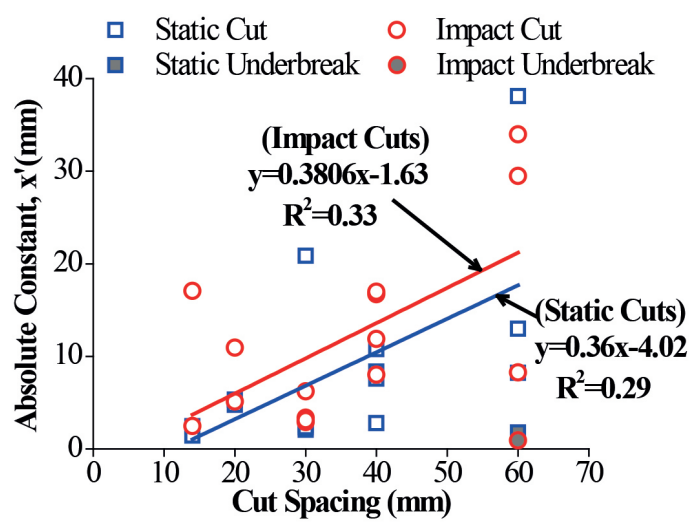

Fig. 11 Effect of cut spacing on the absolute size constant under different load cases

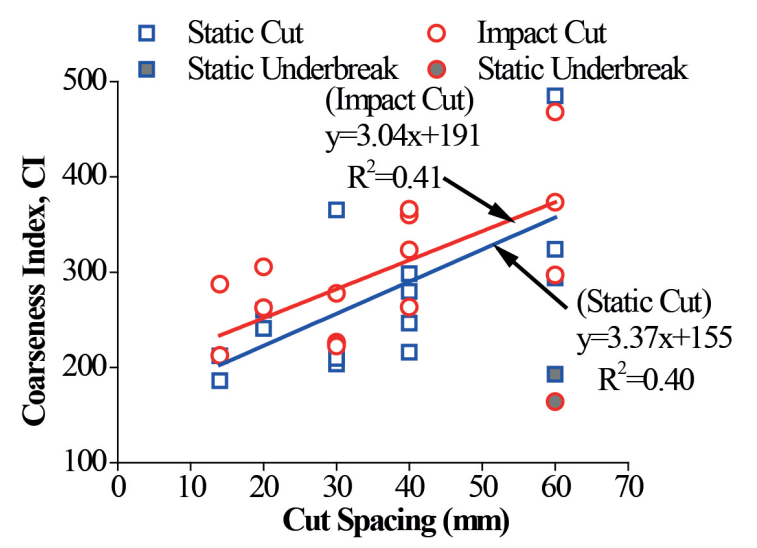

Fig. 12 Effect of cut spacing on the coarseness index under different load cases 


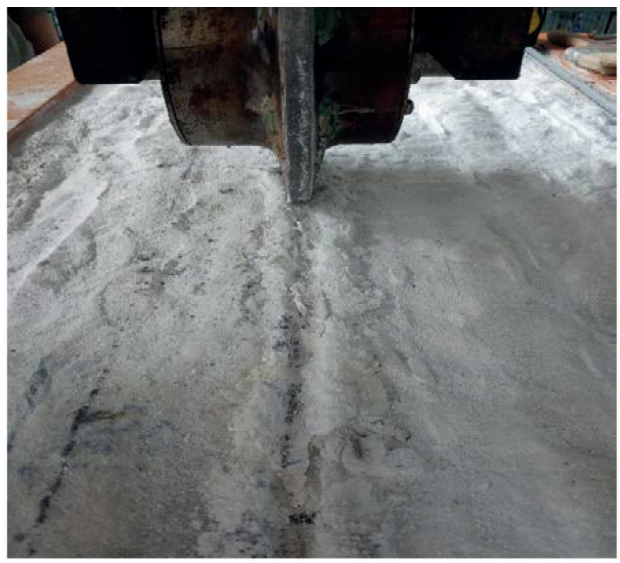

Fig. 13 A typical underbreak cutting model at large $s / p$ ratios under the combination load

\subsection{Relationship between $\mathrm{Cl}, x^{\prime}$ and specific energy}

The specific energy consumption reflects the cutting efficiency. Gertsch et al. [27] reported that the larger the $C I$ values of the rock fragmentation, the higher the corresponding cutting efficiency, which means a lower specific energy value. Fig. 14 and Fig. 15 show the relationship between $C I, x^{\prime}$ and specific energy under different load cases. Because of the low compressive strength and the heterogeneous characteristics of marble samples, there is a wide range in the quality and sizes distribution of the fragments. However, an overall downtrend in SE values can still be seen with the increase of $C I$ and $x^{\prime}$. This shows that the size of broken chips can reflect the energy efficiency in some degree. A large number of large size chips usually mean low $S E$ values. It should be noted that very high $(60 / 2)$ and very low $(14 / 2,20 / 2)$ values of SE exist at the same time at small values of $C I$ and $x^{\prime}$ under static load. At the very wide cut spacing and shallow penetrations, the marble samples are in an underbreak cutting model. A large number of fines and fewer larger chips are produced. The high $S E$ values can be expected. At the same time, some small values of $S E$ appear at the minimum cut spacing and penetration, which are even lower than that under the combination load. This shows that for small penetration and cut spacing, static load cutting can effectively result in a relieved cutting model and the cutting forces are small. While the combination load cut increases the mass of the fragments in a certain extent on the basis of relieved cutting model, the additional impact load leads to a larger $S E$ values. In general, $S E$ under the combination load decreases faster than under the static load.

\subsection{Relationship between $x^{\prime}, \mathrm{Cl}$ and actual production rate}

Because of the high capital cost of mechanical excavation equipment, it is necessary to estimate the productivity and the real cost of the candidate machines accurately. Fig. 16 and Fig. 17 show the relationship between $x^{\prime}, C I$ and actual production rate under different load cases. There is an approximate linear relationship between absolute size constant and the actual production rate. The production rate increases with the increase of $x^{\prime}$. But data fluctuation is relatively large under the combination load condition. Outliers in the combination load cutting paths are attributed to higher and lower $P R_{A}$ data points appear in a number of the same $x^{\prime}$ places. Through analysis of these data points, it can be found that low $P R_{A}$ data points appear in the cutting paths with shallower penetration and smaller cut spacing in contrast to high $P R_{A}$ data points near the same $x^{\prime}$. It indicates that if the rock samples have been in a relieved cutting model under the static load, the applied impact load component will be mainly used for the powderization, and the change of total mass of the fragmentation is not noticeable. Conversely, the application of the impact load can promote the interaction of cracks in the rock under the unrelieved cutting model. A number of large broken chips are produced, resulting in the increase of $P R_{A}$ as well as $x$. To prevent the opposite effect, it's necessary to recognize the field conditions of TBM tunnels before adding the impact load component.

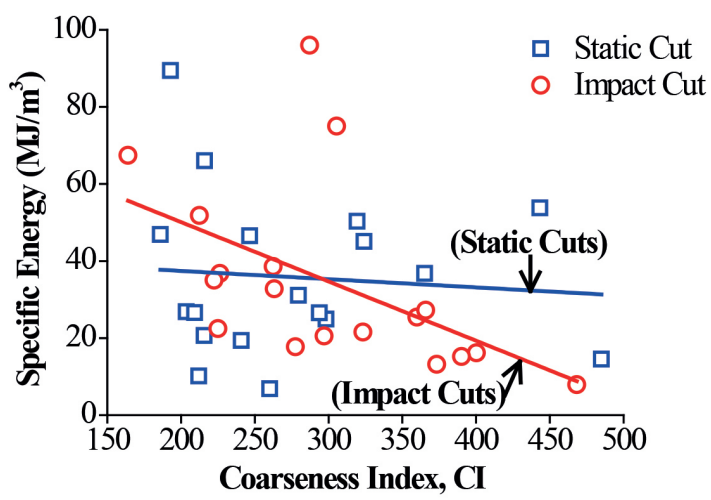

Fig. 14 Relationship between $C I$ and specific energy under different load cases

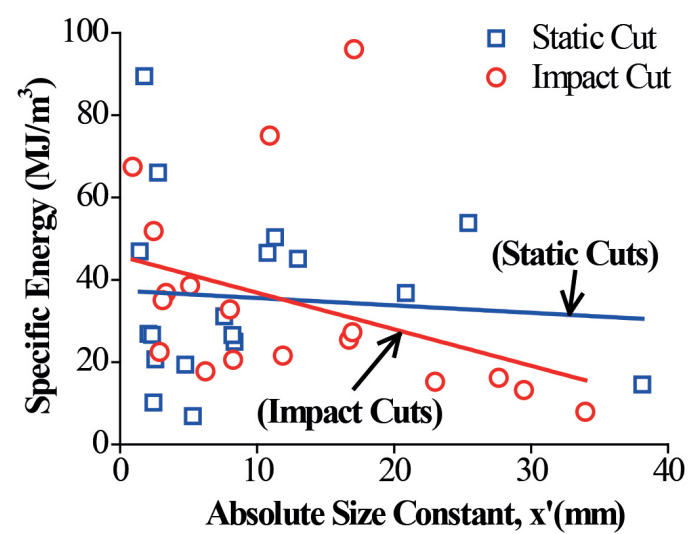

Fig. 15 Relationship between $x^{\prime}$ and specific energy under different load cases

Bakar found the relationship between coarseness index and production rate in the analysis of dry and saturated cutting tests. The fitting curves of $C I$ and $P R_{A}$ under the condition of dry and saturated water are parabolas with an opening [23]. Different from the previous studies, $P R_{A}$ increases linearly with the increase of $C I$, as shown in Fig. 17. It indicates the importance of the most common size of the fragmentation to $P R_{A}$. 
However, the overall difference is not great under the different load cases. The reasons for the different curves of $C I$ and $P R_{A}$ may be due to the different sizes of cutters and the types of rock in two experiments.

The relationship between $C I$ and $P R_{A}$ obtained from the indoor experiments may be different from that in the actual TBM constructions. It's predictable due to the difference in rock type and its anisotropy. Farrokh and Rostami [31] reported that joints with a wide spacing advance the formation of large chips, and joints with a narrow spacing have the opposite effect.

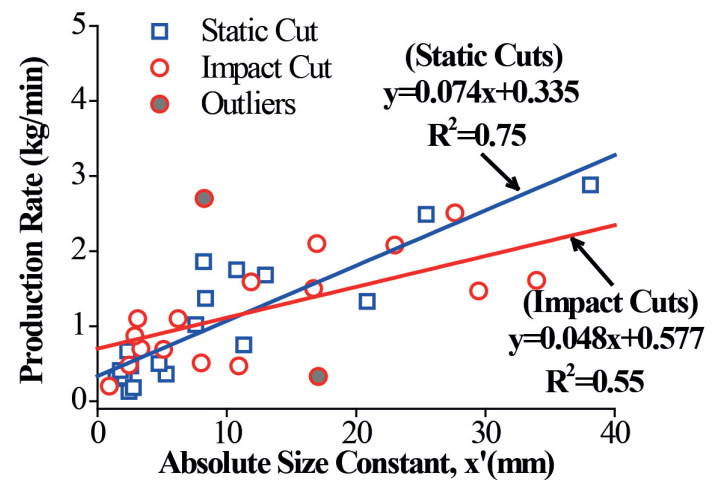

Fig. 16 Relationship between $x^{\prime}$ and actual production rate under different load cases

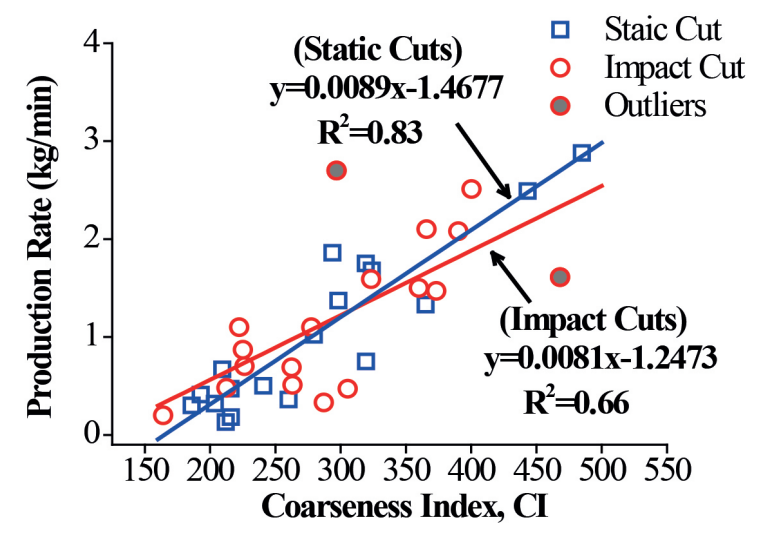

Fig. 17 Relationship between $C I$ and production rate under different load cases

\subsection{Relationship of nominal and actual production rate}

As mentioned in section 4 , the nominal production rate is calculated from the mass of broken chips at the given cutting parameters. The actual production rate is calculated from the broken chips obtained in the experiments. As shown in Fig. 18, a line with a slope of $45^{\circ}$ indicates that the actual production rate is the same as the nominal production rate. Data points above the line indicate an efficient cutting model, in which the cracks inside the rock between the adjacent cutting paths are fully developed and overlapped, resulting in the crushing of the rock below the bottom of the disc cutter. On the contrary, data points below the line indicate an underbreak cutting model. As shown in Fig. 18, when $P R_{N}$ is small, either static or combination load, $P R_{A}$ is greater than $P R_{N}$. When $P R_{N}$ is large, $P R_{A}$ is less than $P R_{N}$. This situation is caused by the conversion of underbreak to overbreak model with the gradual increase of $P R_{N}$. In general, $P R_{A}$ basically floats around the dotted line under the static load and distributes above the dotted line under the combination load. This shows the effect of impact load component on the total mass of fragmentation.

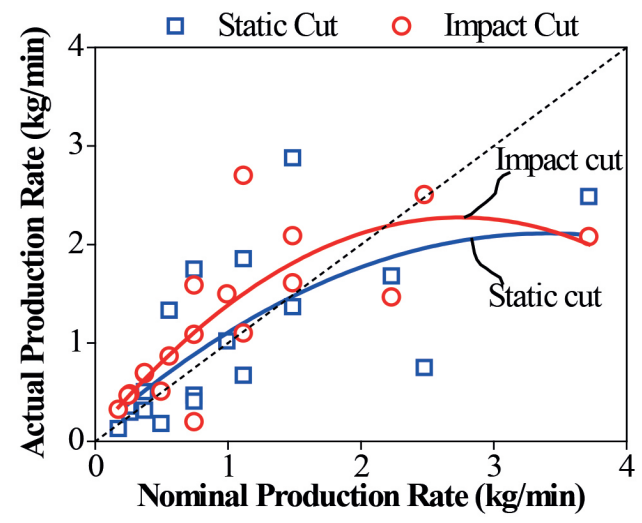

Fig. 18 Relationship between nominal and actual production rate

Make $Q=P R_{A} / P R_{N}$, representing a multiple of $P R_{A}$ relative to $P R_{N}$. Fig. 19 shows the relationship between $Q$ and $s / p$ ratios in different cutting paths. $Q$ rises first and then goes down with the increase of $s / p$ ratios. This conclusion can also be explained by the change of the cutting model. In addition, the $Q$ value under the combination load is obviously larger than that under the static load in the same condition of $s / p$. When $s / p$ is about $15, Q$ reaches its maximum under two load cases. It shows that the impact load can simultaneously promote the development of lateral cracks and radial cracks in the rock. On the one hand, the expansion of lateral cracks can promote the transformation of cutting model. On the other hand, the expansion of the radial crack can break the large area of rock beneath the cutter ring, increasing the total amount of fragments fundamentally. It is worth mentioning that not only $Q$ but also $S E$ should be taken in account while choosing the cutting parameters in the TBM tunnel constructions.

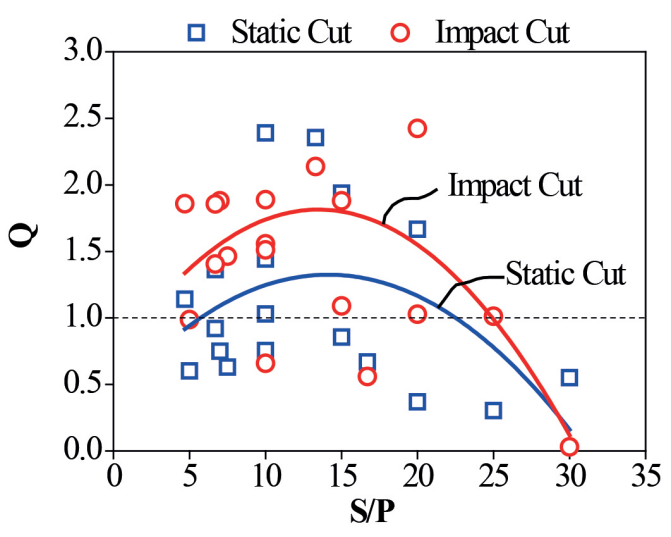

Fig. $19 Q$ variation with $s / p$ 


\section{Conclusions}

In this paper, many marble samples were cut under static load and combination of static and impact load by disc cutters in the multifunctional TBM cutter performance test bench at CSU. The relationship between cutting parameters, specific energy, production rate and the absolute size parameter, coarseness index was studied. The results show:

(1) Increase the cut spacing and penetration leads to an increasing upward trend of $x^{\prime}$ and $C I$. Adding impact load component can further increase $x^{\prime}$ and $C I$ values. It will be a practical way to reduce the number of cutting tools on the cutterhead as well as construction costs.

(2) An overall downtrend in $S E$ with the increase of $C I$ and $x^{\prime}$ can be observed. Besides, $S E$ decreases faster with $C I$ and $x^{\prime}$ under the combination load. On the basis of relieved cutting model, adding the impact load component will increase the SE.

(3) Different from the fitting curves of $C I$ and $P R_{A}$ which are parabolas with an opening under the condition of dry and saturated sandstone, $x^{\prime}$ and $C I$ have an approximate linear relationship with actual production rate. For the rock samples which have been in a relieved cutting mode under the static load, applying impact load component will be mainly used for the powderization and has an opposite effect on the $P R_{A}$.

(4) Both under static and combination load, when $P R_{N}$ is small, $P R_{A}$ is greater than $P R_{N^{\prime}}$. And it has an opposite result when $P R_{N}$ is large. $Q$, which is greater under the combination load, rises first and then goes down with the increase of $s / p$ ratios. Q reaches its maximum when $s / p$ is about 15 under two kinds of load cases. The results can be explained by the change of the cutting model.

\section{Acknowledgement}

This work is supported by the National Natural Science Foundation of China (Granted No. 51475478), the Major State Basic Research Development Program of China (Granted No. 2013CB035401), the Science and Technology Project of Strategic Emerging Industry in Hunan (Granted No. 2015GK1029) and the Central South University Innovation Foundation For Postgraduate (Granted No. 2017zzts405).

\section{References}

[1] Huo, J., Sun, W., Chen, J., Zhang, X. "Disc cutters planet layout design of the full-face rock tunnel boring machine (TBM) based on different layout patterns". Computers and Industrial Engineering, 61(4), pp. 1209-1225. 2011.

https://doi.org/10.1016/j.cie.2011.07.011

[2] Geng, Q., Wei, Z., Meng, H., Chen, Q. "Numerical and experimental research on the rock-breaking process of tunnel boring machine normal disc cutters". Journal of Mechanical Science and Technology, 30(4), pp. 1733-1745. 2016. https://doi.org/10.1007/s12206-016-0329-9

[3] Geng, Q., Wei, Z., Meng, H., Macias, F. J. "Mechanical performance of TBM cutter head in mixed rock ground conditions". Tunnelling and Underground Space Technology, 57, pp. 76-84. 2016. https://doi.org/10.1016/j.tust.2016.02.012
[4] Whittles, D. N., Kingman, S., Lowndes, I., Jackson, K. "Laboratory and numerical investigation into the characteristics of rock fragmentation". Minerals Engineering, 19(14), pp. 1418-1429. 2006.

https://doi.org/10.1016/j.mineng.2006.02.004

[5] Rostami, J., Ozdemir, L. "A new model for performance prediction of hard rock TBMs". In: 1993 Rapid Excavation and Tunneling Conference, [1993 RETC], Boston, Massachusetts, June 13-17, pp. 793-809. 1993.

[6] Balci, C., Tumac, D. "Investigation into the effects of different rocks on rock cuttability by a V-type disccutter". Tunnelling and Underground Space Technology, 30(4), pp. 183-193. 2012.

https://doi.org/10.1016/j.tust.2012.02.018

[7] Xia, Y., Ouyang, T., Xie, L., Tan, Q., Luo, C., Zhao, H., Chen, Z. "The Development of the TBM Disc Cutter Test Device for Cutting Rock". Advanced Materials Research, 889-890, pp. 292-296. 2014. https:// www.scientific.net/AMR.889-890.292

[8] Cho, J. W., Jeon, S., Yu, S. H., Chang, S. H. "Optimum spacing of TBM disccutters: A numerical simulation using the three-dimensional dynamic fracturing method". Tunnelling and Underground Space Technology, 25(3), pp. 230-244. 2010.

https://doi.org/10.1016/j.tust.2009.11.007

[9] Cho, J. W., Jeon, S., Jeong, H. Y., Chang, S. H. "Evaluation of cutting efficiency during TBM disccutter excavation with in a Korean granitic rock using linear-cutting-machine testing and photogrammetric measurement". Tunnelling and Underground Space Technology, 35(4), pp. 37-54. 2013.

https://doi.org/10.1016/j.tust.2012.08.006

[10] Zaré, M., Mikaeil, R. "Optimization of Tunnel Boring Machine (TBM) Disc Cutter Spacing in Jointed Hard Rock Using a Distinct Element Numerical Simulation". Periodica Polytechnica Civil Engineering, 61(1), pp. 56-65. 2016.

https://doi.org/10.3311/PPci.9521

[11] Li, X., Zuo, Y., Ma, C. D. "Failure criterion of strain energy density and catastrophe theory analysis of rock subjected to static-dynamic coupling loading". Chinese Journal of Rock Mechanics and Engineering, 24(16), pp. 2814-2824. 2005.

[12] Gong, F., Li, X., Liu, X., Zhao, J. "Experimental study of dynamic characteristics of sandstone under one-dimensional coupled static and dynamic loads". Chinese Journal of Rock Mechanics and Engineering, 29(10), pp. 2076-2085. 2010.

[13] Zhu, J., Li, X., Gong, F., Wang, S. "Dynamic characteristics and damage model for rock under uniaxial cyclic impact compressive loads". Chinese Journal of Geotechnical Engineering, 35(3), pp. 531-539. 2013.

[14] Zhao, F., Li, X., Feng, T., Deng, X. "A new multifunctional testing device for rock fragmentation". Journal of Central South University of Technology (Natural Science), 35(4), pp. 651-656. 2004.

[15] Zhao, F., Li, X., Feng, T., Xie, S. "Theoretical analysis and experiments of rock fragmentation under coupling dynamic and static loads". Chinese Journal of Rock Mechanics and Engineering, 24(08), pp. 13151320. 2005.

[16] Zhao, F., Wang, H., Peng, Y., Wang, G. "Experimental research on acoustic emission energy and rock crushing effect under static-dynamic coupling loading". Chinese Journal of Rock Mechanics and Engineering, 31(7), pp. 1363-1368. 2012.

[17] Dyskin, A. V. "On the role of stress fluctuations in brittle fracture". International Journal of Fracture, 100(1), pp. 29-53. 1999. https://doi.org/10.1023/A:1018664101433

[18] Li, G., Tao, X. "Testing study on crushing work ratio of rock under dynamic and static load". Chinese Journal of Rock Mechanics and Engineering, 23(14), pp. 2448-2454. 2004. 
[19] Rostami, J. "Performance prediction of hard rock Tunnel Boring Machines (TBMs) in difficult ground". Tunnelling and Underground Space Technology, 57, pp. 173-182. 2016.

https://doi.org/10.1016/j.tust.2016.01.009

[20] Brezáni, I., Zeleňák, F. "Improving the effectivity of work with RosinRammler diagram by using MATLAB R GUI tool". Acta Montanistica Slovaca, 15(2), pp. 152-157. 2010.

[21] Sampson, J. R. "Mineral Processing Design and Operation An Introduction". Chinese Journal of Chemical Engineering, 76(5), pp. 732-732. 2008.

[22] Roxborough, F. F., Rispin, A. "The mechanical cutting characteristics of the Lower Chalk". Tunnels and Tunnelling International, 5(1), pp. 45-67. 1973.

[23] Bakar, M. Z. A., Gertsch, L. S., Rostami, J. "Evaluation of Fragments from Disc Cutting of Dry and Saturated Sandstone". Rock Mechanics and Rock Engineering, 47(5), pp. 1891-1903. 2014. https://doi.org/10.1007/s00603-013-0482-8

[24] Altindag, R. "Estimation of penetration rate in percussive drilling by means of coarseness index and mean particle size". Rock Mechanics and Rock Engineering, 36(4), pp. 323-332. 2003. https://doi.org/10.1007/s00603-003-0002-3

[25] Altindag, R. "Evaluation of drill cuttings in prediction of penetration rate by using coarseness index and mean particle size in percussive drilling". Geotechnical \& Geological Engineering, 22(3), pp. 417-425. 2004. https://doi.org/10.1023/B:GEGE.0000025043.92979.48
[26] Kahraman, S., Develi, K., Yasar, E. "Predicting the penetration rate of percussive blasthole drills using coarseness index and median particle size". CIM Bulletin, 97 (1083), pp. 75. 2004.

[27] Gertsch, R., Gertsch, L. S., Rostami, J. "Disc cutting tests in Colorado Red Granite: implications for TBM performance prediction". International Journal of Rock Mechanics and Mining Sciences, 44, pp. 238 246. 2007.

https://doi.org/10.1016/j.ijrmms.2006.07.007

[28] Jung, H. S., Choi, J. M., Chun, B. S., Park, J. S., Lee, Y. J. "Causes of reduction in shield TBM performance - a case study in Seoul". Tunnelling and Underground Space Technology, 26(3), pp. 453-461. 2011. https://doi.org/10.1016/j.tust.2011.01.001

[29] Cook, N. G. W., Hood, M., Tsai, F. "Observations of Crack Growth in Hard Rock Loaded by an Indenter". International Journal of Rock Mechanics and Mining Sciences and Geomechanics Abstracts, 21(2), pp. 97-107. 1984. https://doi.org/10.1016/0148-9062(84)91177-X

[30] Gupta, A., Yan, D. S. "Particle Size Estimation and Distributions". Chapter 2. In: Mineral Processing Design and Operation, pp. 32-62. Elsevier B.V. 32-62. 2006. https://doi.org/10.1016/B978-044451636-7/50003-6

[31] Farrokh, E., Rostami, J. "Correlation of tunnel convergence with TBM operational parameters and chip size in the Ghomroud tunnel, Iran". Tunnelling and Underground Space Technology, 23(6), pp. 700-710. 2008. https://doi.org/10.1016/j.tust.2008.01.005 